\title{
Functional analysis and comparative genomics of expressed sequence tags from the lycophyte Selaginella moellendorffii Jing-Ke Weng1, Milos Tanurdzic ${ }^{2,3}$ and Clint Chapple*1
}

\author{
Address: ${ }^{1}$ Department of Biochemistry, Purdue University, West Lafayette, IN 47907, USA, ${ }^{2}$ Department of Botany and Plant Pathology, Purdue \\ University, West Lafayette, IN 47907, USA and ${ }^{3}$ current address, Cold Spring Harbor Laboratory, Cold Spring Harbor, NY 11724, USA \\ Email: Jing-Ke Weng - wengj@purdue.edu; Milos Tanurdzic - milos@cshl.edu; Clint Chapple* - chapple@purdue.edu \\ * Corresponding author
}

Published: 06 June 2005

BMC Genomics 2005, 6:85 doi:10.1/86/147|-2/64-6-85

This article is available from: http://www.biomedcentral.com/I47I-2164/6/85

(c) 2005 Weng et al; licensee BioMed Central Ltd.

This is an Open Access article distributed under the terms of the Creative Commons Attribution License (http://creativecommons.org/licenses/by/2.0), which permits unrestricted use, distribution, and reproduction in any medium, provided the original work is properly cited.
Received: 05 March 2005

Accepted: 06 June 2005

\begin{abstract}
Background: The lycophyte Selaginella moellendorffii is a member of one of the oldest lineages of vascular plants on Earth. Fossil records show that the lycophyte clade arose 400 million years ago, 150-200 million years earlier than angiosperms, a group of plants that includes the well-studied flowering plant Arabidopsis thaliana. S. moellendorffii has a genome size of approximately $100 \mathrm{Mbp}$, as small or smaller than that of $A$. thaliana. S. moellendorffii has the potential to provide significant comparative information to better understand the evolution of vascular plants.
\end{abstract}

Results: We sequenced 218I Expressed Sequence Tags (ESTs) from a S. moellendorffii cDNA library. One thousand three hundred and one non-redundant sequences were assembled, containing 291 contigs and 1010 singletons. Approximately $75 \%$ of the ESTs matched proteins in the non-redundant protein database. Among I30I clusters, 343 were categorized according to Gene Ontology (GO) hierarchy and were compared to the GO mapping of $A$. thaliana tentative consensus sequences. We compared S. moellendorffi ESTs to the A. thaliana and Physcomitrella patens EST databases, using the tBLASTX algorithm. Approximately $60 \%$ of the ESTs exhibited similarity with both $A$. thaliana and $P$. patens ESTs; whereas, $13 \%$ and $1 \%$ of the ESTs had exclusive similarity with $A$. thaliana and $P$. patens ESTs, respectively. A substantial proportion of the ESTs (26\%) had no match with $A$. thaliana or $P$. patens ESTs.

Conclusion: We discovered I30I putative unigenes in S. moellendorffii. These results give an initial insight into its transcriptome that will aid in the study of the S. moellendorffii genome in the near future.

\section{Background}

Our understanding of biology has been greatly improved by studying genome structure and gene function of a broad sampling of model organisms such as Mus musculus (mouse), Drosophila melanogaster (fruit fly), Danio rerio (zebrafish), Caenorhabditis elegans (nematode), and Arabidopsis thaliana [1-5]. Comparative genomics has made it clear that orthologs of many proteins that act as signal transduction components, transcriptional regulatory factors, and metabolic enzymes can be identified between and among these model organisms [6]. As a result, the knowledge gained from comparative and evolutionary studies of these species can provide insights into homologous processes in a wide range of other organisms, varying from crop plants to humans [7]. Within plants however, most of the efforts in genomics have been focused on crop 


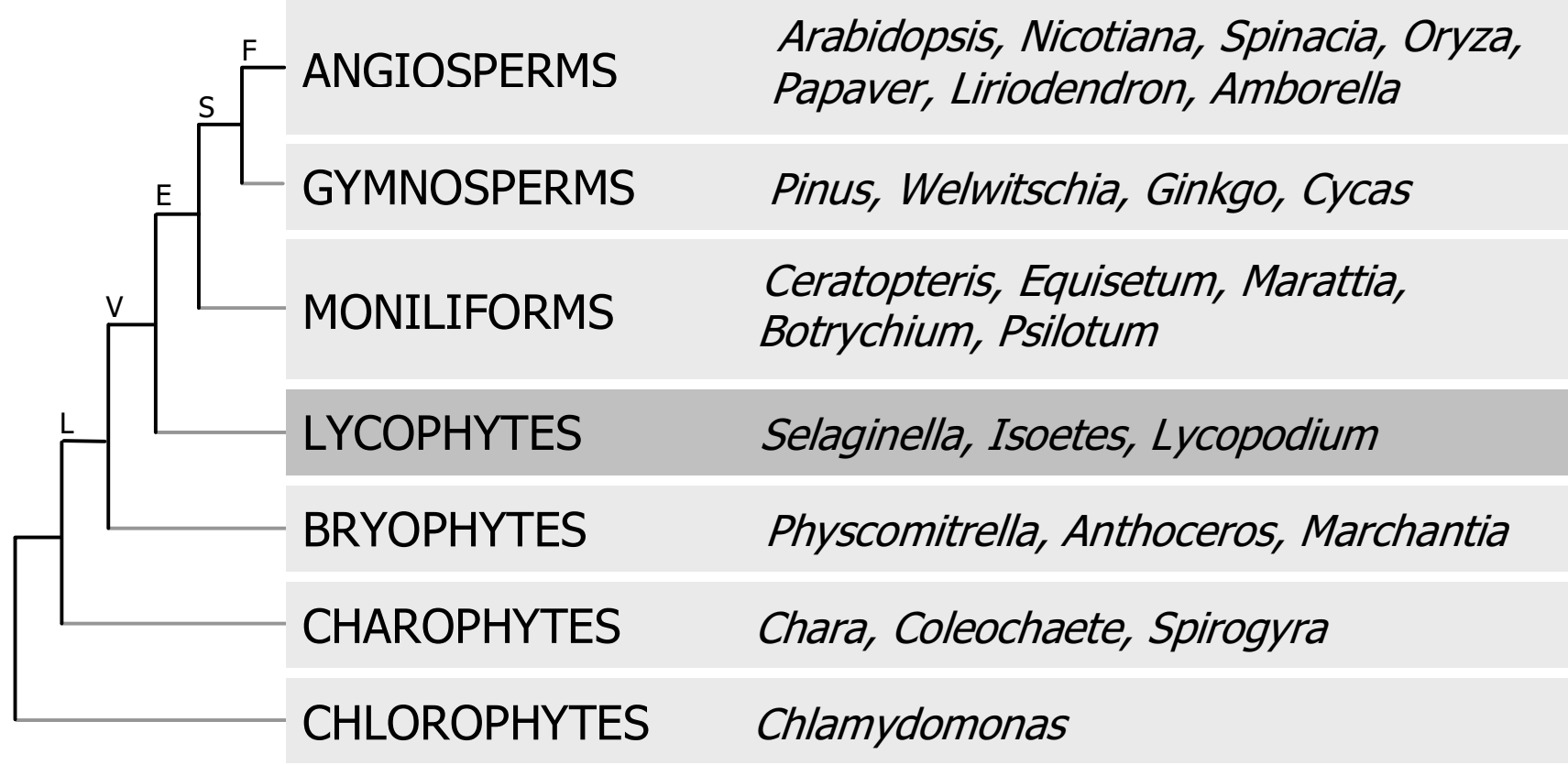

Figure I

A simplified version of the plant phylogenetic tree simplified and condensed from Pryer et al. [I I]. The tree shows that lycophytes (highlighted) diverged from other vascular plant lineages soon after plants colonized the terrestrial environment. Representative species were chosen from sub-clades within the clades listed, and illustrate major developments in plant evolution including the colonization of land (land plants, L), the development of vasculature (vascular plants, V) and true leaves (euphyllophytes, E), and the evolution of flowers (flowering plants, F), and seeds (seed plants, S).

plants or economically important plants such as Oryza sativa (rice), Zea mays (maize), and Lycopersicon esculentum (tomato) [8-10]. Thus, coupled with the sequencing of the A. thaliana genome, these efforts have provided data on only a single branch of the plant evolutionary tree, namely members of the Monocotyledonae and Dicotyledonae, collectively termed the angiosperms and commonly known as flowering plants. As a result, the community of plant scientists has little sequence data on other plant lineages that could provide insights into common mechanisms of how plants develop and survive in a terrestrial environment, nor do they have any kind of evolutionary benchmarks that might reveal how angiosperms have come to dominate most world ecosystems [11].

Clear evidence for the existence of angiosperms is present in the fossil record of the lower Cretaceous (140 million years ago), and some evidence suggests their existence 60 million years earlier, around the same time that conifers and ginkgos arose [12]. In contrast, fossil evidence for the lycophytes is found in strata dated to approximately 420 million years ago [13]. Thus, this clade diverged very early from the lineage that led to all other vascular plants (Fig- ure 1), and has existed on earth over twice as long as plants that are the most common subjects of current laboratory and agricultural research. As such, the study of lycophytes may provide novel insights into plant biology that would not be provided by research that focuses only on flowering plants.

Selaginella is an extant genus of the lycophyte clade. It is sometimes referred to as a 'seed-free' plant to highlight the fact that it has not evolved flowers and seeds in the time since its divergence from other plant lineages. It has a number of characteristics that would make its study convenient for, and valuable to, the plant biology community $[11,14]$. For example, like many other species of Selaginella, S. moellendorffii (Figure 2) is a small diploid plant that can be easily grown in the laboratory. Further, it has an approximate genome size of $100 \mathrm{Mbp}$ [14], smaller than that of $A$. thaliana, and among the smallest published genome sizes for 'seed-free' genera. Because of these attributes, S. moellendorffii was recently chosen as one of the non-crop plants for BAC library construction in a NSF funded Green Plant BAC library Project [15]. More importantly, the Department of Energy Joint Genome Institute 


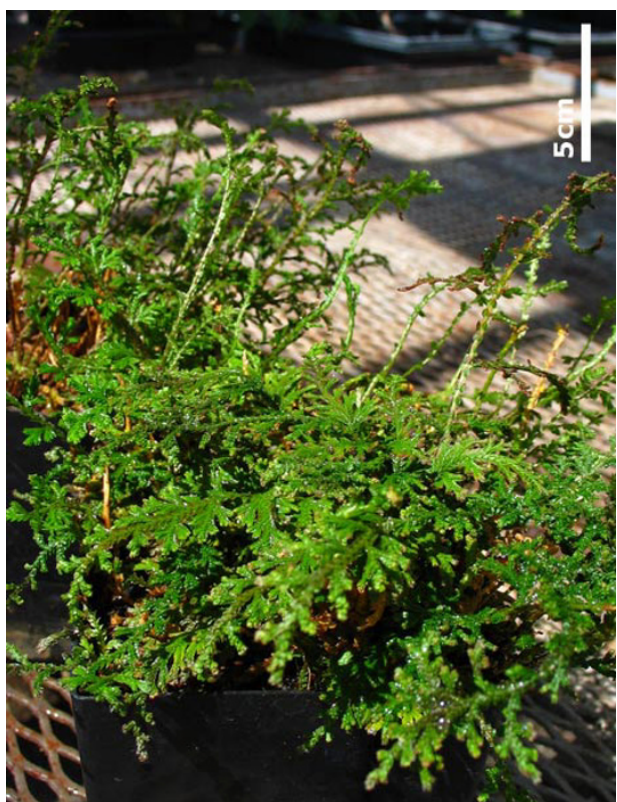

(a)

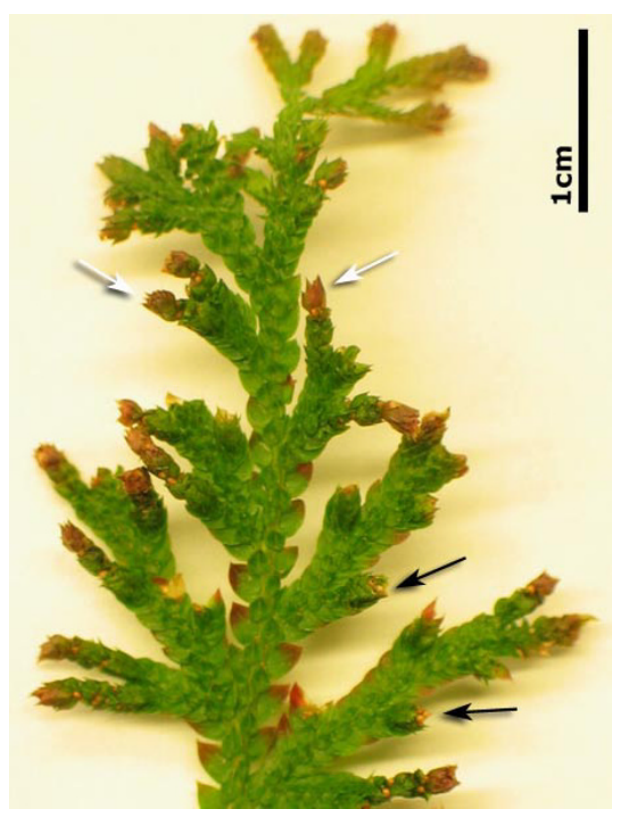

(b)

\section{Figure 2}

The morphology of $S$. moellendorffii. (a) A greenhouse grown S. moellendorffii. (b) A close up of an aerial branch of $S$. moellendorffii indicating the bulbils (white arrows) that can be used for clonal propagation and sporangia (black arrows) containing microspores and megaspores for sexual propagation.
(JGI) has officially announced that it will sequence the $S$. moellendorffii genome [16], making this species a target of extreme interest for research into comparative plant genomics, biochemistry, and development.

Expressed sequence tag (EST) sequencing has been used as an efficient and economical approach for large-scale gene discovery [17]. It has also successfully provided frameworks for many genome projects $[18,19]$. Recently, a large number of ESTs have been generated from various plant species and deposited in GenBank, including both model and crop plants like $A$. thaliana, rice, wheat, and maize as well as species representative of clades other than angiosperms, such as gymnosperms, cycads, and mosses [20-23]. Although over 1000 ESTs from another Selaginella species $S$. lepidophylla, also known as the resurrection plant, have also been deposited in GenBank [20], no manuscript has been published reporting on their analysis. In this paper, we describe 2181 ESTs generated from a S. moellendorffii cDNA library. These ESTs were assembled into 1301 clusters, annotated using the BLASTX algorithm, surveyed for their abundance within the dataset, and classified into functional groups according to the Gene Ontology (GO) hierarchy. Finally, a comparative genomics approach was used for comparing $S$. moellendorffii ESTs with those of A. thaliana and Physcomitrella patens to look for genes unique to $S$. moellendorffii.

\section{Results and Discussion}

\section{Generation of S. moellendorffii cDNA library and ESTs}

To gain a broad coverage of $S$. moellendorffii transcripts, we collected and pooled whole S. moellendorffii plants for mRNA extraction and subsequent cDNA library construction. To enrich for full-length cDNA clones, doublestranded cDNA was size-fractionated before cloning. Based upon the average insert sizes of 35 cDNA clones chosen at random from the library, we estimate that the cDNA library has an average insert size of 850 bp. 2304 clones were sequenced from the $5^{\prime}$ end of the cDNAs, which generated 2181 vector-trimmed EST sequences with an average sequencing read length of $640 \mathrm{bp}$.

\section{Assembly of S. moellendorffii ESTs}

To identify overlapping EST sequences, reduce sequencing error and produce non-redundant EST data for further functional annotation and comparative analysis, 2181 ESTs were assembled into clusters through stackPACK v2.2 clustering system [24]. Based upon regions of nucleotide identity, EST sequences were merged into contiguous consensus sequences (contigs). One thousand three hundred and one non-redundant EST clusters, putatively regarded as unigenes, were generated, consisting of 291 contigs and 1010 singletons. The cluster size varied from one to 105 copies of any given EST (Figure 3). Manual inspection of the assembled ESTs identified 10 clusters 


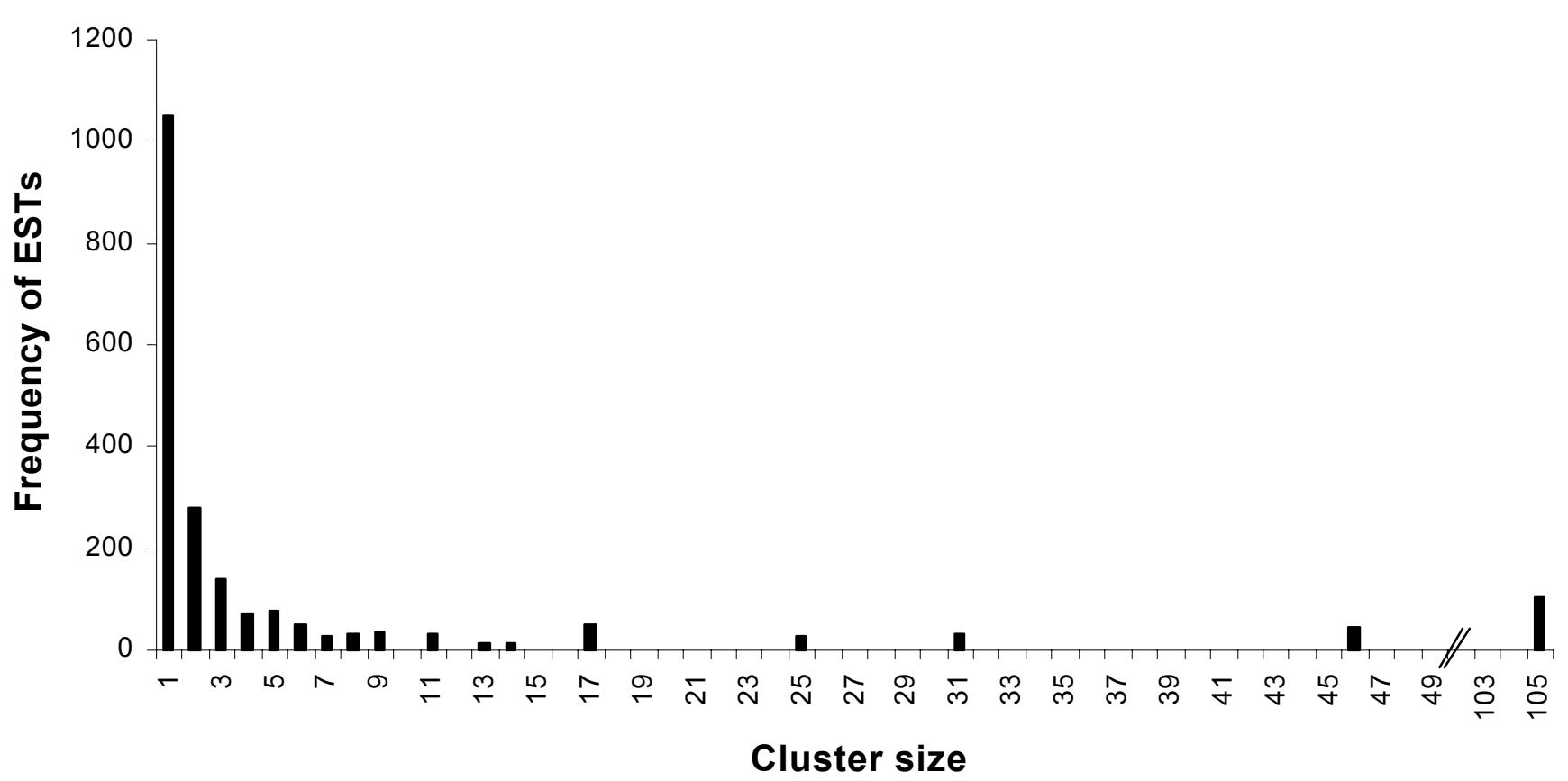

Figure 3

Distribution of S. moellendorffii ESTs by cluster size. ESTs were clustered into putative unigene sets using StackPack v. 2.2 , and the number of cluster members of each size category was plotted relative to their abundance within the EST collection.

counted as unigenes that may actually represent non-overlapping sequence reads from cDNAs corresponding to four single genes. As an example, three unigenes were found to be best aligned to three different regions of the same protein in a BLASTX analysis (described in the following paragraph), suggesting we lack a complete transcript for their accurate assembly. Conversely, we also found that some clustered ESTs did not necessarily have identical sequences within their overlapping regions. In most of the cases, regions of sequence disagreement within the clusters tend to appear towards the ends of the EST reads, which is likely to be caused by errors generated during sequencing. In some other cases, it may due to failure to discriminate between gene family members during clustering, or allelic diversity in S. moellendorffii.

\section{Annotation of S. moellendorffii ESTs}

To annotate $S$. moellendorffii ESTs, the 1301 putative unigenes were translated dynamically in all 6 reading frames and searched for homology against the NCBI non-redundant (nr) protein database using BLASTX [25]. BLASTX hits with E-values less than $10^{-5}$ were taken to be significant. Among 1301 unigenes, 962 (74\%) had BLASTX hits in the nr database, while the remaining 339 (26\%) had hits with E-values greater than $10^{-5}$ or no hit. When a less permissive cutoff E-value of $10^{-10}$ was adopted, the numbers of unigenes with BLASTX hits and without BLASTX hits changed slightly to 891 (68\%) and 410 (32\%) respectively. Our dataset showed that the inferred translation products of most $S$. moellendorffii ESTs appear to be similar to proteins in other organisms but that there was also a percentage of ESTs that represented potential Selaginellaor lycophyte-specific genes. Interestingly, 15 ESTs had at least their top five BLASTX hits from non-plant organisms, including six from bacteria or cyanobacteria (SmoC1_02_N06, SmoC-1_01_C17, SmoC-1_02_B19, SmoC1_06_K12,SmoC-1_cn167, SmoC-1_03_D21), two from fungi (SmoC-1_06_O23, SmoC-1_02_H20), one from an insect (SmoC-1_06_K02), three from nematodes (SmoC1_04_D10, SmoC-1_02_L08, SmoC-1_cn108), one from fish (SmoC-1_04_F24), and two from mammals (SmoC1_02_H05, SmoC-1_03_F21). These data suggest that homologs have either not yet been identified or are absent from other plant lineages, although in one case (SmoC1_06_O23), a more distantly related A. thaliana gene was returned by BLASTX, and in a further three cases, BLASTN 
Table I: The most abundantly represented ESTs in the S. moellendorffii cDNA library.

\begin{tabular}{|c|c|c|c|c|c|}
\hline & \multirow[t]{2}{*}{ Cluster } & \multirow{2}{*}{$\begin{array}{l}\text { Number } \\
\text { of ESTs }\end{array}$} & \multicolumn{3}{|c|}{ Top BLASTX hit in non-redundant protein database } \\
\hline & & & $\begin{array}{l}\text { Accession } \\
\text { Number }\end{array}$ & Best Identity Description & E-value \\
\hline 1 & SmoC-I_cn 126 & 105 & - & Novel & - \\
\hline 2 & SmoC-I_cn 125 & 46 & - & Novel & - \\
\hline 3 & SmoC-I_cn0I8 & 31 & SP:PI603I & Ribulose bisphosphate carboxylase small subunit) [Larix laricina] & $8 \mathrm{E}-5 \mathrm{I}$ \\
\hline 4 & SmoC-I_cn $|2|$ & 25 & SP:P04669 & Ferredoxin, chloroplast precursor [Silene latifolia subsp. alba] & $2 \mathrm{E}-26$ \\
\hline 5 & SmoC-I_cnl06 & 17 & PIR:SI6294 & chlorophyll a/b-binding protein [Lycopersicon esculentum] & $9 \mathrm{E}-99$ \\
\hline 6 & SmoC-I_cnl07 & 17 & GB:AAM46780 & latex plastidic aldolase-like protein [Hevea brasiliensis] & IE-164 \\
\hline 7 & SmoC-I_cnI7I & 17 & PIR:S3I863 & chlorophyll a/b-binding protein [Pinus sylvestris] & IE-106 \\
\hline 8 & SmoC-I_cnOII & 14 & GB:AAC78I07 & photosystem-I H subunit GOS5 [Oryza sativa] & $8 \mathrm{E}-30$ \\
\hline 9 & SmoC-I_cn233 & 13 & SP:Q9SXW9 & Plastocyanin, chloroplast precursor [Physcomitrella patens] & $2 \mathrm{E}-37$ \\
\hline 10 & SmoC-I_cn025 & 11 & SP:P5III8 & glutamine synthetase cytosolic isoenzyme I [Vitis vinifera] & $1 E-152$ \\
\hline 11 & SmoC-I_cn089 & 11 & GB:AAGI 7036 & S-adenosylmethionine synthetase [Pinus contorta] & $7 E-17$ \\
\hline 12 & SmoC-I_cnl95 & 11 & SP:PII432 & Early light-induced protein, chloroplast precursor (ELIP) [Pisum sativum] & IE-32 \\
\hline 13 & SmoC-I_cn023 & 9 & SP:P82977 & Subtilisin-chymotrypsin inhibitor [Triticum aestivum] & $4 \mathrm{E}-\mathrm{II}$ \\
\hline 14 & SmoC-I_cnl45 & 9 & - & Novel & - \\
\hline 15 & SmoC-I_cnl79 & 9 & SP:P3036I & $\begin{array}{l}\text { Cytochrome } \mathrm{B}_{6}-\mathrm{F} \text { complex iron-sulfur subunit I, chloroplast precursor [Nicotiana } \\
\text { tabacum] }\end{array}$ & $3 \mathrm{E}-74$ \\
\hline 16 & SmoC-I_cnl89 & 9 & - & Novel & - \\
\hline 17 & SmoC-I_cn006 & 8 & GB:AAG59875 & PSII subunit PsbW [Physcomitrella patens] & $5 \mathrm{E}-13$ \\
\hline 18 & SmoC-I_cn078 & 8 & SP:O48560 & Catalase 3 [Glycine max] & 0 \\
\hline 19 & SmoC-I_cn2II & 8 & SP:P23993 & Photosystem I reaction center subunit XI, chloroplast precursor [Hordeum vulgare] & $2 \mathrm{E}-55$ \\
\hline 20 & SmoC-I_cn226 & 8 & PDB:IEKJA & Carbonic Anhydrase [Pisum Sativum] & $2 \mathrm{E}-63$ \\
\hline 21 & SmoC-I_cn019 & 7 & REF:NP_I 175963 & photosystem I reaction center subunit $\mathrm{V}$, chloroplast, [Arabidopsis thaliana] & $2 \mathrm{E}-34$ \\
\hline 22 & SmoC-I_cnl08 & 7 & PIR:T235। 2 & hypothetical protein $\mathrm{K} 08 \mathrm{HI} 0.2 \mathrm{a}$ [Caenorhabditis elegans] & $\mathrm{IE}-12$ \\
\hline 23 & SmoC-I_cn2I5 & 7 & GB:AAB88617 & ubiquitin conjugating enzyme [Zea mays] & $3 \mathrm{E}-82$ \\
\hline 24 & SmoC-I_cn218 & 7 & SP:P27494 & Chlorophyll a-b binding protein 36 , chloroplast precursor [Nicotiana tabacum] & $\mid E-127$ \\
\hline 25 & SmoC-I_cn0I3 & 6 & PIR:T0647I & core protein [Pisum sativum] & $\mathrm{IE}-20$ \\
\hline 26 & SmoC-I_cn016 & 6 & SP:Q9SLQ8 & Oxygen-evolving enhancer protein 2, chloroplast precursor [Cucumis sativus] & IE-79 \\
\hline 27 & SmoC-I_cn033 & 6 & GB:AAM970II & expressed protein [Arabidopsis thaliana] & $6 \mathrm{E}-40$ \\
\hline 28 & SmoC-I_cn I36 & 6 & GB:AAO49652 & photosystem I-N subunit [Phaseolus vulgaris] & $2 \mathrm{E}-37$ \\
\hline 29 & SmoC-I_cnl39 & 6 & DBJ:BAC66946 & chloroplastic iron superoxide dismutase [Barbula unguiculata] & $3 \mathrm{E}-69$ \\
\hline 30 & SmoC-I_cn 180 & 6 & EMB:CAB7| 293 & chloroplast ferredoxin-NADP+ oxidoreductase precursor [Capsicum annuum] & IE-139 \\
\hline 31 & SmoC-I_cn208 & 6 & SP:P54773 & Photosystem II 22 kDa protein, chloroplast precursor [Lycopersicon esculentum] & $5 \mathrm{E}-61$ \\
\hline 32 & SmoC-I_cn250 & 6 & - & Novel & - \\
\hline
\end{tabular}

Non-redundant protein database includes all non-redundant GenBank CDS translations (GB)+ RefSeq Proteins (REF) +PDB + SwissProt (SP) + PIR + PRF. The identities of ESTs were putatively described by the top BLASTX hit (with lowest E-value) of the assembled EST contigs.

analysis of the EST-others database identified potential homologs in $P$. patens (SmoC-1_02_N06, SmoC1_06_K12) and S. lepidophylla (SmoC-1_cn167).

\section{Highly represented S. moellendorffii ESTs}

EST copy number can be used to approximate gene expression levels in an organism, although there are artifacts of cDNA library construction that may limit or over-represent certain transcripts [26]. Table 1 summarizes the first 32 most abundantly represented transcripts in the $S$. moellendorffii EST collection, having six or more EST copies in each cluster, with their identities putatively assigned by BLASTX analysis of the assembled contigs. As expected, a large number of the $S$. moellendorffii ESTs are photosyn- thesis-related genes, with 19 clusters containing 213 ESTs ( $9 \%$ of total sequenced ESTs) corresponding to genes involved in photosynthesis. There were seven clusters matching to core proteins of photosynthesis reaction centers, including four subunits of photosystem I (PSI-G, PSI$\mathrm{H}$, PSI-L, PSI-N), and three photosystem II proteins (PsbW, OEC23, CP22). There were four contigs corresponding to light-harvesting chlorophyll a/b-binding proteins, including one early light-induced protein. We also found ESTs for the RuBisCO small subunit, carbonic anhydrase, plastocyanin, one subunit of cytochrome $b_{6} f$ complex, ferredoxin and ferredoxin/NADP oxidoreductase, proteins involved in carbon fixation and photosynthetic electron transport. There were two putative anti- 
oxidative proteins found within S. moellendorffii ESTs: chloroplastic iron superoxide dismutase and catalase, presumably required for the decomposition of superoxide and hydrogen peroxide $[27,28]$. The BLASTX results show that all of these highly expressed $S$. moellendorffii photosynthetic genes had homologs in A. thaliana genome, consistent with previous observation that the photosynthesis machinery has been highly conserved throughout plant evolution.

Three highly expressed S. moellendorffii transcripts corresponded to genes encoding enzymes of metabolism, including an aldolase-like protein, a putative glutamine synthetase cytosolic isoenzyme involved in nitrogen assimilation $[29,30]$, and a putative S-adenosylmethionine synthetase required for the synthesis of the major methyl group donor involved in the methylation of a variety of biomolecules ranging from histones to secondary metabolites, and for the biosynthesis of ethylene [31,32].

Other relatively abundant ESTs included one encoding a putative subtilisin-chymotrypsin inhibitor, exhibiting $49 \%$ amino acid sequence identity with the wheat subtilisin-chymotrypsin inhibitor, which may play a role in plant defense by inhibiting the serine proteinases of pathogens [33]. Two transcripts that matched an A. thaliana expressed protein and Pisum sativum core protein may function as membrane channel proteins. Interestingly, one highly expressed EST matched with an E-value of $10^{-}$ 12 a C. elegans protein of unknown function, and is only more distantly related to an $A$. thaliana late embryogenesis abundant protein.

There were five highly expressed ESTs that did not yield significant matches using BLASTX $\left(\mathrm{E}>10^{-5}\right)$. These are putative Selaginella-specific genes and may encode proteins with functions unique to Selaginella or lycophytes. The first two highly expressed ESTs in this project, represented by clusters SmoC1_cn126 and SmoC1_cn125, had 105 and 46 copies in their clusters respectively, but returned no BLASTX hits with the nr protein database or BLASTN hits with the NCBI EST-others database. To determine whether these sequences represented bona fide Selaginella genes, we amplified the corresponding sequences by PCR using genomic DNA as a template (data not shown). Both sequences amplified successfully, and both had introns, indicating that they were not derived from DNA contamination from prokaryotic symbionts. The rational translation of SmoC1_cn126 contig contains a three repeats of the motif "XXXGXXTCDKCAQTGVCTCGKN", which aligns with similar cysteine-rich motifs in proteins with epidermal growth factor repeats. Using a low BLASTX stringency $(\mathrm{E}=0.002)$, SmoC1_cn125 matched to a Cynodon dactylon metallothionein-like protein (GB:AAS88721.1, 75\% identical within a 20 amino acid motif). The other three highly expressed S. moellendorffii specific ESTs lack hints for functional annotation. The biological function of the proteins encoded by these genes, and the question of whether high transcript abundance is predictive of high protein expression will be a matter for future investigation.

\section{Functional categorization of S. moellendorffii ESTs}

The most sensitive method to find new members of known gene families among EST sequences is to search for homology of the translated ESTs to motifs extracted from a multiple alignment of known gene family members [18]. To functionally categorize S. moellendorffii ESTs using motif homology searches, we translated the 1301 unigenes in six reading frames and imported them into InterProScan [34], which aligned 491 clusters to InterPro entries $\left(\mathrm{E}<10^{-5}\right)$. Mapping of InterPro entries to GO [35], assigned 343 out of 491 InterPro hits with 562 GO accession numbers. The 562 accession numbers further generated 964 individual GO mappings in the three major ontologies (biological processes, molecular functions and cellular components) [36]. The apparent discrepancies between these values arises from the fact that not all InterPro hits had available GO accession numbers associated with them, one InterProScan entry could be assigned to more than one GO accession numbers, and one GO accession number could be mapped under multiple parental categories [37].

Tables 2 and Figure 4 summarize the GO assignment of $S$. moellendorffii ESTs in terms of biological processes, molecular functions and cellular components, covering a broad range of the GO functional categories. Using the downloaded A. thaliana GO assignments from the TIGR A. thaliana Gene Index [38,39], we compared the distribution of GO categories between S. moellendorffii ESTs and A. thaliana tentative consensus sequences (TCs). Table 3 shows that the distribution patterns of GO assignments of $S$. moellendorffii and A. thaliana transcripts were generally similar, with a few exceptions in some categories. Besides the true differences in functional distribution of unigenes, some of the differences could be due to the difference in EST data sources between these two species. For example, in terms of biological processes, A. thaliana has a higher percentage in 'response to stimulus and stress' and 'development' than S. moellendorffii. Considering that among the A. thaliana ESTs in the TIGR database, some were generated from plants at specific developmental stages or from plants exposed to specific biotic or abiotic stimuli, it is very likely that ESTs from orthologs of these genes would be missing from the S. moellendorffii ESTs which were generated from normal mature plants.

The current GO annotations for plants are based solely on the annotated proteins of $A$. thaliana and O. sativa, both of 
Table 2: The GO categorization of S. moellendorffii ESTs by biological process, molecular function, and cellular component.

\begin{tabular}{|c|c|c|c|}
\hline & Gene Ontology term & Representation & Representation percentage \\
\hline \multirow[t]{14}{*}{ Biological process } & Metabolism & 312 & $74 \%$ \\
\hline & Biosynthesis & 64 & $15 \%$ \\
\hline & Protein metabolism & 57 & $14 \%$ \\
\hline & Catabolism & 22 & $5 \%$ \\
\hline & Nucleic acid metabolism & 20 & $5 \%$ \\
\hline & Cell growth and/or maintenance & 53 & $13 \%$ \\
\hline & Transport & 44 & $10 \%$ \\
\hline & Response to stimulus and stress & 19 & $5 \%$ \\
\hline & Photosynthesis & 16 & $4 \%$ \\
\hline & Cell communication & 15 & $4 \%$ \\
\hline & Signal transduction & 12 & $3 \%$ \\
\hline & Homeostasis & 3 & $1 \%$ \\
\hline & Development & I & $<1 \%$ \\
\hline & Cell death & I & $<1 \%$ \\
\hline \multirow[t]{18}{*}{ Molecular function } & Catalytic activity & 132 & $36 \%$ \\
\hline & Hydrolase activity & 36 & $10 \%$ \\
\hline & Transferase activity & 25 & $7 \%$ \\
\hline & Oxidoreductase activity & 22 & $6 \%$ \\
\hline & Kinase activity & 12 & $3 \%$ \\
\hline & Binding & 107 & $29 \%$ \\
\hline & Nucleotide binding & 65 & $18 \%$ \\
\hline & Metal ion binding & 20 & $5 \%$ \\
\hline & Transporter activity & 64 & $18 \%$ \\
\hline & Electron transporter activity & 16 & $4 \%$ \\
\hline & Carrier activity & 12 & $3 \%$ \\
\hline & Structural molecule activity & 40 & $11 \%$ \\
\hline & Translation regulator activity & 10 & $3 \%$ \\
\hline & Signal transducer activity & 4 & $1 \%$ \\
\hline & Chaperone activity & 3 & $1 \%$ \\
\hline & Enzyme regulator activity & 2 & $1 \%$ \\
\hline & Motor activity & I & $<1 \%$ \\
\hline & Transcription regulator activity & I & $<1 \%$ \\
\hline \multirow[t]{2}{*}{ Cellular component } & Intracellular & 135 & $75 \%$ \\
\hline & Membrane & 45 & $25 \%$ \\
\hline
\end{tabular}

Note that one gene product may be assigned to more than one GO terms, and one children term can fit into multiple parental categories. The representation means the number of non-redundant ESTs that can be mapped to a certain GO term. The representation percentage is based on the total number of GO mappings in each of the three major ontologies (biological process: 420 , molecular function: 364 , cellular component: 180).

which are angiosperms. Since the lycophyte clade diverged from other plant lineages 400 million years ago, and 200 million years before angiosperms, it is perhaps to be expected that a large proportion of $S$. moellendorffii genes could not be accurately assigned to GO categories in the database containing only angiosperm gene entries. We expect that the representation of plant species other than angiosperms will certainly benefit resources as InterPro and in turn will lead to further resolution within GO.

\section{Comparative genomics of S. moellendorffii ESTs}

One important objective of comparative genomics is to trace gene evolution including the emergence, development, and loss of orthologous genes in different organisms over evolutionary time [40]. To survey the $S$. moellendorffii ESTs in an evolutionary context, we used the
S. moellendorffii unigene sequences as queries to search for homologous sequences in the $A$. thaliana and $P$. patens EST databases using tBLASTX algorithm (cut off E-value $=10^{-}$ $\left.{ }^{6}\right)$. There were two reasons that we chose $A$. thaliana and $P$. patens ESTs as tBLASTX databases. First, $A$. thaliana and $P$. patens are representatives of the most diverged lineages of land plants, namely angiosperms and bryophytes. They flank Selaginella in the plant phylogenetic tree, and last shared a common ancestor over 400 million years ago [23], thus providing ample opportunity for the evolutionary divergence of individual genes and gene families. Second, the large quantities of $A$. thaliana and $P$. patens ESTs in GenBank (472,278 and 104,027 respectively) provide a substantial coverage of the transcriptome in these two species. Using them as BLAST databases makes it possible 
Table 3: Comparison of GO assignments between A. thaliana ESTs and S. moellendorffii ESTs.

\begin{tabular}{|c|c|c|c|}
\hline \multirow[t]{2}{*}{ Gene Ontology term } & \multirow[t]{2}{*}{ Categories } & \multicolumn{2}{|c|}{ Representation percentage } \\
\hline & & S. moellendorffii & A. thaliana \\
\hline \multirow[t]{8}{*}{ Biological process } & Metabolism & $74 \%$ & $39 \%$ \\
\hline & Cell growth and/or maintenance & $13 \%$ & $13 \%$ \\
\hline & Response to stimulus and stress & $5 \%$ & $16 \%$ \\
\hline & Photosynthesis & $4 \%$ & $<1 \%$ \\
\hline & Cell communication & $4 \%$ & $6 \%$ \\
\hline & Homeostasis & $1 \%$ & $1 \%$ \\
\hline & Development & $<1 \%$ & $6 \%$ \\
\hline & Cell death & $<1 \%$ & $1 \%$ \\
\hline \multirow[t]{10}{*}{ Molecular function } & Catalytic activity & $36 \%$ & $41 \%$ \\
\hline & Binding & $29 \%$ & $32 \%$ \\
\hline & Transporter activity & $18 \%$ & $8 \%$ \\
\hline & Structural molecule activity & $11 \%$ & $2 \%$ \\
\hline & Translation regulator activity & $3 \%$ & $1 \%$ \\
\hline & Signal transducer activity & $1 \%$ & $1 \%$ \\
\hline & Chaperone activity & $1 \%$ & $2 \%$ \\
\hline & Enzyme regulator activity & $1 \%$ & $1 \%$ \\
\hline & Motor activity & $<1 \%$ & $1 \%$ \\
\hline & Transcription regulator activity & $<1 \%$ & $7 \%$ \\
\hline \multirow[t]{2}{*}{ Cellular component } & Intracellular & $75 \%$ & $70 \%$ \\
\hline & Membrane & $25 \%$ & $19 \%$ \\
\hline
\end{tabular}

The GO assignments for A. thaliana ESTs were obtained from TIGR [38]. The percentage of GO assignments for A. thaliana was calculated based on the total numbers of GO mappings in each of the three major ontologies with the number of unknown terms deducted from them (biological process: 20185 , molecular function: 23680 , cellular component: 6309 ). The functional categories present in $A$. thaliana but not in S. moellendorffii were not listed in the table.

to do a relatively comprehensive genomic analysis even in the absence of the full genome sequence of $P$. patens.

Figure 5 summarizes the distribution of $S$. moellendorffii ESTs by tBLASTX results. Among 1301 non-redundant $S$. moellendorffii ESTs, 788 (61\%) ESTs had homology with both $A$. thaliana and $P$. patens ESTs. These ESTs probably identify non-dispensable genes, which tend to be evolutionarily conserved in all land plants [41]. $168(13 \%)$ ESTs had exclusive similarity with A. thaliana ESTs, and may represent the genes that evolved in land plants after the divergence of bryophytes, or those that were lost from the genomes of mosses. Table 4 shows the top $20 \mathrm{~S}$. moellendorffii EST tBLASTX hits for A. thaliana ESTs that were not present within the $P$. patens EST database ranked by tBLASTX E-values. Among these, it is possible to identify candidates that might have contributed to the success of vascular plants, including those involved in functions such as lignification (SmoC-1_05_G17) [42], cell division control (SmoC-1_01_E02) [43], intracellular transport (SmoC-1_02_C05 and SmoC-1_05_G03) [44,45], responses to sulfur starvation (SmoC-1_03_C14) [46], dehydration (SmoC-1_06_M11), and viral infection (SmoC-1_06_P21) [47]. Only 8 (1\%) S. moellendorffii ESTs had similarity only with $P$. patens ESTs. These ESTs may represent genes that arose early in plant evolution but were lost later after the divergence of the lycophytes. It should be noted, however, that all eight of these ESTs had relative low tBLASTX score (E-value around $10^{-10}$ ), limiting our certainty that the homologous ESTs in $P$. patens are true orthologs. Finally, there were 337 (26\%) ESTs that had no tBLASTX match in the $A$. thaliana and $P$. patens EST databases. These ESTs may be Selaginella-specific genes, possibly having evolved only in lycophytes after their divergence from other lineages or having arisen after the divergence of bryophytes and later being lost in euphyllophytes.

\section{Conclusion}

We sequenced 2181 ESTs from the lycophyte S. moellendorffii, putatively representing 1301 unigenes. Our data showed that a large proportion of the genes had homologous genes in the well-studied model plant $A$. thaliana and other plant species. By browsing the putative functional annotations of these ESTs, researchers will be able to choose S. moellendorffii genes of interest and compare them to their othologs in other species. We also found a substantial number of putative Selaginella-specific genes that do not share similarity with known genes, with some of them even representing very highly expressed genes. 
(a)

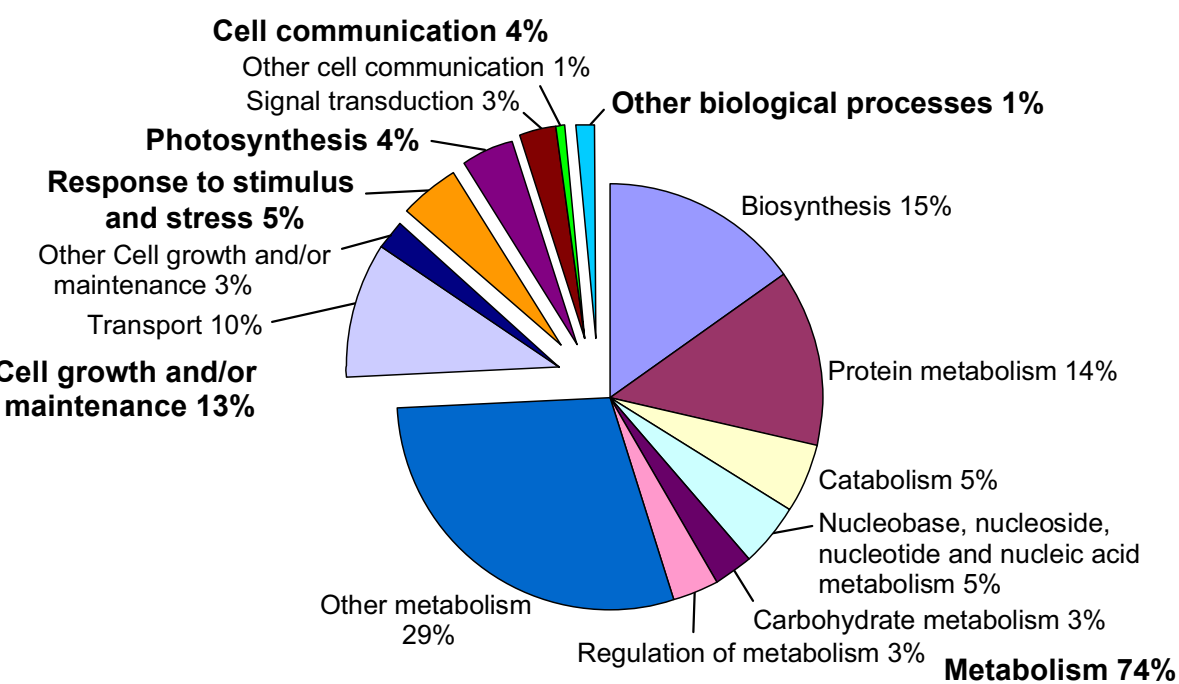

(b)

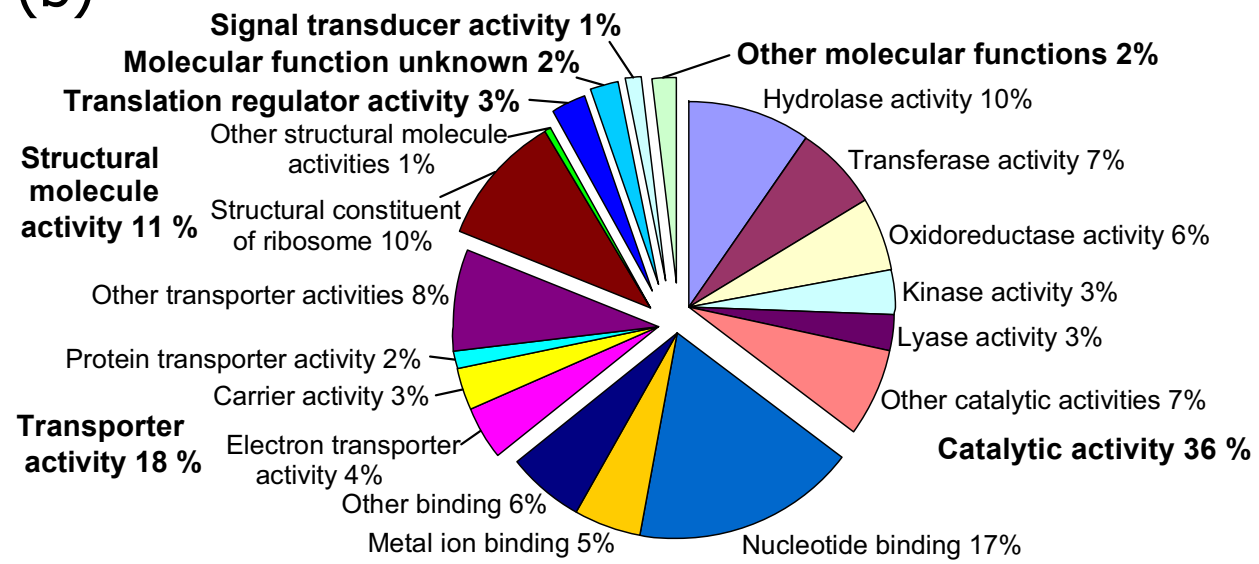

Binding $29 \%$

(c)

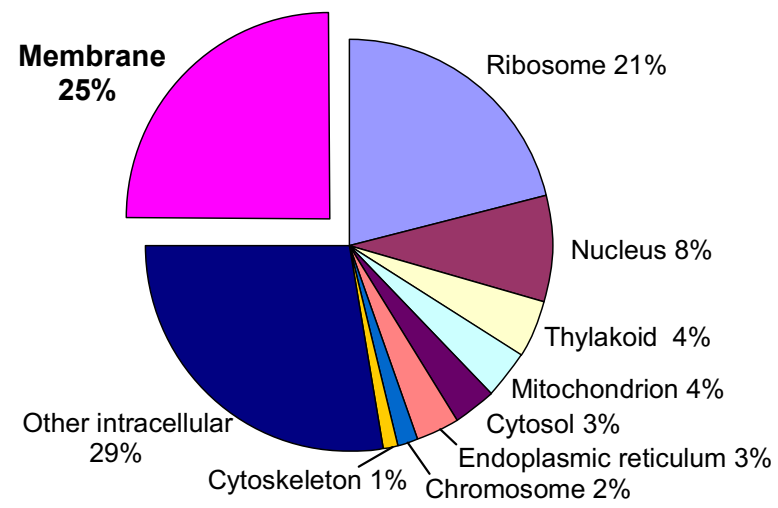

Intracellular

$75 \%$

Figure 4

Representations of Gene Ontology (GO) mapping results for S. moellendorffii non-redundant ESTs. (a) Biological process (b) Molecular function (c) Cellular component. 


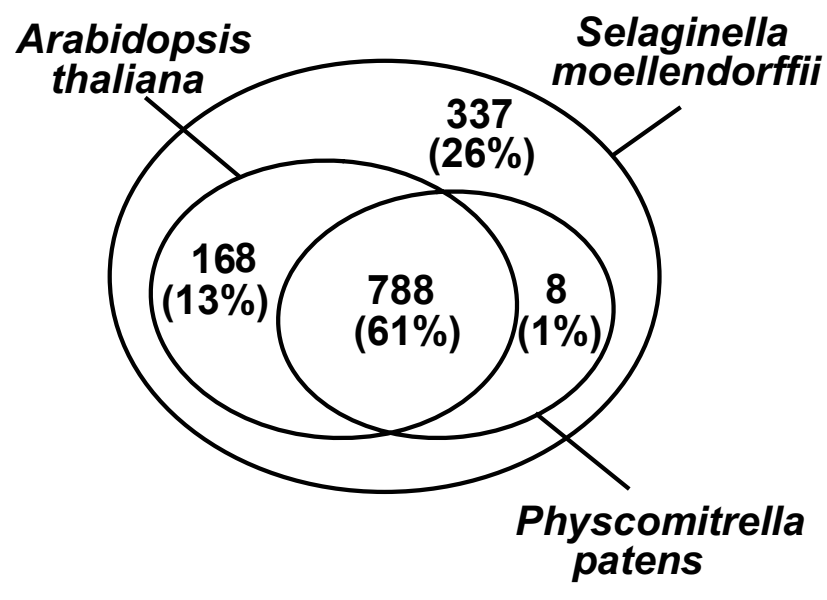

Figure 5

A Venn diagram showing the distribution of S. moellendorffii EST tBLASTX matches by databases. The I30I translated S. moellendorffii non-redundant ESTs were used as queries in homology searches against $A$. thaliana and $P$. patens EST databases, respectively. The two inner circles contain the numbers and percentages of S. moellendorffii ESTs that share tBLASTX similarity with $A$. thaliana or $P$. patens ESTs. The region between inner circles and outer circle represents $S$. moellendorffii ESTs without tBLASTX matches.

Considering the complexity of the plant kingdom and a time span more than 150 million years between the divergences of lycophytes and angiosperms, it will not be surprising to identify gene functions in $S$. moellendorffii that are not present in $A$. thaliana. When the draft genome sequence of $S$. moellendorffii is completed and released, this EST resource will also play an important role in the mapping and annotation of the genome. As a member of a clade that arose after the bryophytes and before all other vascular plants, $S$. moellendorffii will provide new opportunities in studying plant evolution, particularly those adaptations relating to fundamental traits that facilitated the transition of green plants to the land, such as lignification in vascular plants, root/stem/leaf organography, complex patterns of sporophyte branching, and the elaboration of reproductive structures.

\section{Methods}

\section{Plant material and cDNA library Construction}

S. moellendorffii was obtained from Plant Delights Nursery (Raleigh, NC). Plants were grown at $23^{\circ} \mathrm{C}$ in a greenhouse with a photoperiod of $16 \mathrm{~h}$ light $/ 8 \mathrm{~h}$ dark. The cDNA library used in this study was made from RNA extracted from pooled tissue including stems, microphylls, strobilis, and rhizophores of $S$. moellendorffii plants. Briefly, fresh tissue was ground in liquid nitrogen and total RNA was extracted using the RNeasy Max Kit (QIAGEN, Valencia, CA), treated with RNase-free DNase, and precipitated in $2 \mathrm{M}$ lithium chloride. Poly $\mathrm{A}+\mathrm{RNA}$ was isolated from total RNA using the Dynabeads mRNA Purification Kit (Dynal Biotech, Brown Deer, WI). The cDNA library was constructed from $1 \mu \mathrm{g}$ mRNA using the Creator Smart cDNA Library Construction Kit (CLONTECH, Palo Alto, CA). After first-strand synthesis, the full length double stranded cDNAs were synthesized by primer-extension. Full length double stranded cDNAs were digested with $S f i$ I and size fractionated using a CHROMA SPIN-400 column (CLONTECH, Palo Alto, CA). cDNA-containing fractions were pooled, and ethanol precipitated. The cDNAs were then cloned into pDNR-LIB at $S f i$ I site, and electroporated into E. coli DH10B cells (Invitrogen, Carlsbad, CA). The library had an un-amplified titer of $1.6 \times 10^{6} \mathrm{col}-$ ony-forming units $\mathrm{mL}^{-1}$ and a total complexity of $3.2 \times 10^{6}$ colonies. To estimate the average insert size of the library, plasmid DNAs were extracted from 35 randomly picked clones from the library, digested with $S f i$ I, and analyzed by agarose gel electrophoresis.

\section{EST sequencing and dbEST submission}

18,432 colonies from un-amplified $S$. moellendorffii cDNA library were arrayed into 48 384-well plates using Q-Pix multifunction colony picker (Genetix). Plasmid DNA was isolated from 2304 clones picked from the first six 384well plates. Sequences of cDNAs were determined from their 5 ' end by conventional procedures using the big-dye terminators on the ABI 3730xl DNA analyzer (Applied Biosystems, Foster City, CA) at the Purdue Genomics Center using T7-ZL (5'-TAATACGACTCACTATAGGG-3') as the 5'-sequencing primer. The vector sequence was trimmed from the original EST sequences resulting in 2181 sequences. The 2181 ESTs have been submitted to GenBank dbEST under the accession numbers DN837577 to DN839757 [20].

\section{EST clustering and homology search}

2181 EST sequences were imported into the stackPACK v2.2 clustering system (Electric Genetics, Reston, VA) through WebPipe for clustering with default setting, and contig consensus sequences were generated from the clusters. One thousand three hundred and one non-redundant EST sequences were exported through WebReport in FASTA format. BLASTX analyses using the nr database were performed on the 1301 unigene sequences, using Evalue of $10^{-5}$ as a cutoff threshold. The complete BLASTX annotation of $1301 \mathrm{~S}$. moellendorffii unigenes can be viewed at [48].

\section{Functional categorization of ESTs}

To search for functional protein domains of translated ESTs, 1301 unigene sequences were merged into one FASTA file and imported into InterProScan, which was 
Table 4: Top 20 S. moellendorffii EST tBLASTX hits for A. thaliana ESTs that are not present within the $P$. patens EST database.

\begin{tabular}{|c|c|c|c|c|}
\hline & Non-redundant EST & tBLASTX E-value & Best BLASTX Descriptor in A. thaliana & Accession Number \\
\hline 1 & SmoC-I_0I_H05 & IE- 107 & expressed protein & REF:NP_194688 \\
\hline 2 & SmoC-I_02_C05 & IE-99 & oligopeptide transporter OPT family protein & REF:NP_1928I5 \\
\hline 3 & SmoC-I_0I_L23 & $2 \mathrm{E}-99$ & putative Mg-protoporphyrin IX chelatase & REF:NP_196867 \\
\hline 4 & SmoC-I_05_GI7 & $2 \mathrm{E}-99$ & putative caffeoyl-CoA 3-O-methyltransferase & REF:NP_195131 \\
\hline 5 & SmoC-I_05_KI3 & $5 \mathrm{E}-90$ & chloroplast membrane protein (ALBINO3) & REF:NP_I 180446 \\
\hline 6 & SmoC-I_0I_E02 & IE-89 & cullin family protein & REF:NP_567243 \\
\hline 7 & SmoC-I_05_G03 & 7E-87 & putative UDP-galactose/UDP-glucose transporter & REF:NP_563949 \\
\hline 8 & SmoC-I_05_II9 & $6 \mathrm{E}-86$ & expressed protein & REF:NP_566060 \\
\hline 9 & SmoC-I_02_NI5 & $9 \mathrm{E}-86$ & nicotinate phosphoribosyltransferase family protein & REF:NP_I79923 \\
\hline 10 & SmoC-I_03_10I & $7 E-80$ & glycoside hydrolase family 77 protein & REF:NP_181616 \\
\hline 11 & SmoC-I_cn293 & $4 \mathrm{E}-77$ & amine oxidase family protein & REF:NP_I81830 \\
\hline 12 & SmoC-I_03_C24 & $9 \mathrm{E}-73$ & uridylyltransferase-related protein & REF:NP_564010 \\
\hline 13 & SmoC-I_02_PI4 & $5 \mathrm{E}-70$ & expressed protein & REF:NP_I 199542 \\
\hline 14 & SmoC-I_06_P2I & $2 \mathrm{E}-69$ & RNase $L$ inhibitor protein-related & REF:NP_196569 \\
\hline 15 & SmoC-I_05_GI0 & $3 \mathrm{E}-69$ & expressed protein & REF:NP_191746 \\
\hline 16 & SmoC-I_03_CI4 & IE-66 & putative isoflavone reductase & REF:NP_565107 \\
\hline 17 & SmoC-I_03_N06 & $6 \mathrm{E}-65$ & transducin / WD-40 repeat family protein & REF:NP_190148 \\
\hline 18 & SmoC-I_06_MII & IE-63 & dehydration stress-induced protein & GB:AAM 62648 \\
\hline 19 & SmoC-I_06_B20 & IE-60 & putative membrane protein & REF:NP_849987 \\
\hline 20 & SmoC-I_05_O2I & $2 \mathrm{E}-60$ & paired amphipathic helix repeat-containing protein & REF:NP_I8678| \\
\hline
\end{tabular}

The tBLASTX E-value of an EST varies with its BLASTX E-value in a small range (e.g. SmoC-I_0I_H05 has a tBLASTX E-value of IE-I07 against its homologous A. thaliana EST and a BLASTX E-value of 2E-94 against the translated full length $A$. thaliana cDNA.). The homology ranking was based on the tBLASTX E-value. The identities of ESTs were putatively described by the $A$. thaliana protein with the lowest E-value in the BLASTX analysis.

run on a local SUN unix server. BlastProDom, Coil, FPrintScan, HMMPIR, HMMPfam, HMMSmart, HMMTigr, ProfileScan, ScanRegExp, and Seg superfamily were selected as the database methods. All the sequences were translated in six reading frames and aligned to the entries in the selected databases. EST clusters which had positive InterProScan hits $\left(\mathrm{E}<10^{-5}\right)$ were automatically assigned InterPro accession numbers. According to the mapping of InterPro entries to GO [35], GO accession numbers were assigned to EST clusters, which were used to classify ESTs into functional groups by molecular function, cellular component, and biological process. In comparison of the distribution of GO categories between $S$. moellendorffii ESTs and A. thaliana TCs, the GO assignments for A. thaliana ESTs were obtained from TIGR [38]. The Complete Interpro assignment and GO mapping of $S$. moellendorffii ESTs can be accessed in the supplemental data (see Additional file: 1 ).

\section{Comparison of S. moellendorffii ESTs to A. thaliana and P. patens ESTs}

472,278 A. thaliana ESTs and 104,027 P. patens ESTs retrieved from GenBank by searching 'Arabidopsis / Physcomitrella and gbdiv est' in NCBI Entrez [25] were saved to a local server. The 1301 S. moellendorffii unigenes were translated in six reading frames and searched for homology against the six-frame translations of $A$. thaliana ESTs and $P$. patens ESTs respectively using the BLAST algorithm.
An E-value of $10^{-6}$ was set as stringency threshold. The complete result of $S$. moellendorffii unigenes tBLASTX against $A$. thaliana and $P$. patens ESTs can be viewed at [48].

\section{Genomic PCR}

To amplify the genomic sequences of the two most highly expressed ESTs (SmoC1_cn126 and SmoC1_cn125) in S. moellendorffii, PCR was performed using genomic DNA extracted from $50 \mathrm{mg}$ fresh tissue of $S$. moellendorffii as described previously [49] as template and two pairs of PCR primers designed from their EST contig sequences: CC1170 (5'-CGAGCTCGTAGTGATAGTGTC -3') and CC1171 (5'-AACCATAGGAGAGGAAGACC-3') for SmoC1_cn126; CC1228 (5'-ATAGCTTAGCTGCTTTCTTCTC-3') and CC1229 (5'-ATACTACTCATGTCGCAGCTC -3') for SmoC1_cn125. PCR was performed using an initial 2 min denaturation at $94^{\circ} \mathrm{C}$, followed by 25 cycles, each consisting of a $0.5 \mathrm{~min}$ denaturation at $94^{\circ} \mathrm{C}$, a 0.5 min annealing at $50^{\circ} \mathrm{C}$, and a 1 min extension at $72^{\circ} \mathrm{C}$. These 25 cycles were followed by a 5 min extension at $72{ }^{\circ} \mathrm{C}$. PCR products were purified using QIAquick PCR Purification Kit (QIAGEN) and sequenced at Purdue Genomics Center.

\section{Authors' contributions}

JKW constructed the S. moellendorffii cDNA library, participated in the EST sequencing, carried out the bioinfomatic 
analysis of the ESTs, and performed the genomic PCR for two transcripts. MT participated in the S. moellendorffii cDNA library construction and provided comments on the manuscript. CC conceived the study and coordinated work. JKW and CC wrote the article. All authors read and approved the final manuscript.

\section{Additional material}

\section{Additional file 1}

The Complete Interpro assignment and GO mapping of S. moellendorffii ESTs, Excel file.

Click here for file

[http://www.biomedcentral.com/content/supplementary/14712164-6-85-s1.xls]

\section{Acknowledgements}

This research was supported by a grant from the National Science Foundation to C.C. and a pilot project grant from the Department of Biochemistry, Purdue University. This is journal paper number 2005- 17677 from the Purdue University Agricultural Experiment Station. We thank Dr. Jo Ann Banks for critically reading the manuscript.

\section{References}

I. Mouse Genome Sequencing Consortium: Initial sequencing and comparative analysis of the mouse genome. Nature 2002, 420:520-562.

2. Adams MD, Celniker SE, Holt RA, Evans CA, Gocayne JD, Amanatides PG, Scherer SE, Li PW, Hoskins RA, Galle RF, et al.: The genome sequence of Drosophila melanogaster. Science 2000, 287:2185-2195.

3. Grunwald DJ, Eisen JS: Headwaters of the zebrafish - emergence of a new model vertebrate. Nat Rev Genet 2002:717-724.

4. The $C$. elegans Sequencing Consortium: Genome Sequence of the Nematode $C$. elegans: A Platform for Investigating Biology. Science 1998, 282:2012-2018.

5. Arabidopsis Genome Initiative: Analysis of the genome sequence of the flowering plant Arabidopsis thaliana. Nature 2000, 408:796-8I5.

6. O'Brien SJ, Menotti-Raymond M, Murphy WJ, Nash WG, Wienberg J, Stanyon R, Copeland NG, Jenkins NA, Womack JE, Marshall Graves JA: The Promise of Comparative Genomics in Mammals. Science 1999, 286:458-48I.

7. Miller W, Makova KD, Nekrutenko A, Hardison RC: Comparative genomics. Annu Rev Genomics Hum Genet 2004, 5: I 5-56.

8. Yu J, Hu S, Wang J, Wong GK, Li S, Liu B, Deng Y, Dai L, Zhou Y, Zhang $X$, et al:: $A$ draft sequence of the rice genome (Oryza sativa $L$. ssp. indica). Science 2002, 296:79-92.

9. Martienssen RA, Rabinowicz PD, O'Shaughnessy A, McCombie WR: Sequencing the maize genome. Curr Opin Plant Biol 2004, 7:102-107.

10. Tanksley SD, Ganal MW, Prince JP, de Vicente MC, Bonierbale MW, Broun P, Fulton TM, Giovannoni Jj, Grandillo S, Martin GB, et al.: High density molecular linkage maps of the tomato and potato genomes. Genetics 1992, I 32: I I I I-60.

II. Pryer KM, Schneider H, Zimmer EA, Banks JA: Deciding among green plants for whole genome studies. Trends in Plant Sci 2002, 7:550-554

12. Stewart WN, Rothwell GW: Paleobotany and the evolution of plants 2 nd edition. Cambridge University Press, Cambridge, UK; 1993.

13. Kenrick $P$, Crane PR: The origin and early evolution of plants on land. Nature 2003, 389:33-39.

14. Wang W, Tanurdzic M, Luo M, Sisneros N, Kim HR, Weng JK, Kudrna D, Mueller C, Arumuganathan K, Carlson J, et al.: Construction of a bacterial artificial chromosome library from the spikemoss
Selaginella moellendorffii: A new resource for plant comparative genomics. BMC Plant Biol 2005, 5: 10.

15. The Green Plant BAC Library Project [http://www.green
[ bac.org]

16. JGI Approved Community Sequencing Program Projects for 2005 [http://www.jgi.doe.gov/sequencing/cspseqplans.html]

17. Whitfield CW, Band MR, Bonaldo MF, Kumar CG, Liu L, Pardinas JR, Robertson HM, Soares MB, Robinson GE: Annotated expressed sequence tags and cDNA microarrays for studies of brain and behavior in the honey bee. Genome Res 2002, 12:555-566.

18. Jongeneel CV: Searching the expressed sequence tag (EST) databases: panning for genes. Brief Bioinform 2000, 1:76-92.

19. Adams MD, Kelley JM, Gocayne JD, Dubnick M, Polymeropoulos MH, Xiao H, Merril CR, Wu A, Olde B, Moreno RF, et al.: Complementary DNA sequencing: expressed sequence tags and human genome project. Science 1991, 252:165I-1656.

20. NCBI expressed sequence tag database [http:// www.ncbi.nlm.nih.gov/dbEST]

21. Kirst M, Johnson AF, Baucom C, Ulrich E, Hubbard K, Staggs R, Paule C, Retzel E, Whetten R, Sederoff R: Apparent homology of expressed genes from wood-forming tissues of loblolly pine (Pinus taeda L.) with Arabidopsis thaliana. Proc Natl Acad Sci USA 2003, 100:7383-7388.

22. Brenner ED, Stevenson DW, McCombie RW, Katari MS, Rudd SA, Mayer KF, Palenchar PM, Runko SJ, Twigg RW, Dai G, et al:: Expressed sequence tag analysis in Cycas, the most primitive living seed plant. Genome Biol 2003, 4:R78.

23. Nishiyama T, Fujita T, Shin-I T, Seki M, Nishide H, Uchiyama I, Kamiya A, Carninci P, Hayashizaki Y, Shinozaki K, et al:: Comparative genomics of Physcomitrella patens gametophytic transcriptome and Arabidopsis thaliana: implication for land plant evolution. Proc Natl Acad Sci USA 2003, 100:8007-80 I 2.

24. stackPACK [http://www.egenetics.com/stackpack.html]

25. NCBI [http://www.ncbi.nlm.nih.gov]

26. McCarter JP, Mitreva MD, Martin J, Dante M, Wylie T, Rao U, Pape $D$, Bowers $Y$, Theising B, Murphy CV, et al.: Analysis and functional classification of transcripts from the nematode Meloidogyne incognita. Genome Biol 2003, 4:R26.

27. McKersie BD, Murnaghan J, Jones KS, Bowley SR: Iron-superoxide dismutase expression in transgenic alfalfa increases winter survival without a detectable increase in photosynthetic oxidative stress tolerance. Plant Physiol 2000, I 22:1427-1438.

28. Fita I, Rossmann MG: The active center of catalase. J Mol Biol 1985, 185:21-37.

29. Mann AF, Fentem PA, Stewart GR: Identification of two forms of glutamine synthetase in barley (Hordeum Vulgare). Biochem Biophys Res Commun 1979, 88:515-521.

30. Oliveira IC, Coruzzi GM: Carbon and Amino Acids Reciprocally Modulate the Expression of Glutamine Synthetase in Arabidopsis. Plant Physiol 1999, 121:301-310.

31. Yang SF, Hoffman NE: Ethylene biosynthesis and its regulation in higher plants. Annu Rev Plant Physiol 1984, 35:155-189.

32. Lamblin F, Saladin G, Dehorter B, Cronier D, Grenier E, Lacoux J, Bruyant P, Laine E, Chabbert B, Girault F, et al.: Overexpression of a heterologous sam gene encoding S-adenosylmethionine synthetase in flax (Linum usitatissimum) cells: Consequences on methylation of lignin precursors and pectins. Physiol Plant 200I, I I 2:223-232.

33. Poerio E, Gennaro SD, Maro AD, Farisei F, Ferranti P, Parente A: Primary structure and reactive site of a novel wheat proteinase inhibitor of subtilisin and chymotrypsin. Biol Chem 2003, 384:295-304.

34. Mulder NJ, Apweiler R, Attwood TK, Bairoch A, Barrell D, Bateman A, Binns D, Biswas M, Bradley P, Bork P, et al.: The InterPro Database, 2003 brings increased coverage and new features. Nuc Acids Res 2003, 31 1:315-318.

35. Mapping of InterPro entries to GO [http://www.geneontol ogy.org/external2go/interpro2go]

36. Gene Ontology Consortium [http://www.geneontology.org]

37. Gene Ontology Consortium: Creating the gene ontology resource: design and implementation. Genome Res 200I, II:I425-1433.

38. TIGR Arabidopsis Gene Index [http://www.tigr.org/tigr-scripts/ tgi/ $T$ index.cgi?species=arab]

39. Quackenbush J, Cho J, Lee D, Liang F, Holt I, Karamycheva S, Parvizi B, Pertea G, Sultana R, White J: The TIGR Gene Indices: analysis 
of gene transcript sequences in highly sampled eukaryotic species. Nucleic Acids Res 200I, 29:159-164.

40. Mirkin BG, Fenner TI, Galperin MY, Koonin EV: Algorithms for computing parsimonious evolutionary scenarios for genome evolution, the last universal common ancestor and dominance of horizontal gene transfer in the evolution of prokaryotes. BMC Evol Biol 2003, 3:2.

4I. Jordan IK, Rogozin IB, Wolf YI, Koonin EV: Essential genes are more evolutionarily conserved than are nonessential genes in bacteria. Genome Res 2002, I 2:962-968.

42. Guo D, Chen F, Inoue K, Blount JW, Dixon RA: Downregulation of caffeic acid 3-O-methyltransferase and caffeoyl CoA 3-Omethyltransferase in transgenic alfalfa. impacts on lignin structure and implications for the biosynthesis of $\mathbf{G}$ and $\mathbf{S}$ lignin. Plant Cell 200I, I3:73-88.

43. Kipreos ET, Lander LE, Wing JP, He WW, Hedgecock EM: cul-I is required for cell cycle exit in $C$. elegans and identifies a novel gene family. Cell 1996, 85:829-839.

44. Koh S, Wiles AM, Sharp JS, Naider FR, Becker JM, Stacey G: An oligopeptide transporter gene family in Arabidopsis. Plant Physiol 2002, I 28:2। -29.

45. Norambuena L, Marchant L, Berninsone P, Hirschberg CB, Silva $H$, Orellana A: Transport of UDP-galactose in plants: Identification and functional characterization of AtUTr I, an Arabidopsis thaliana UDP-galactose/UDP-glucose transporter. J Biol Chem 2002, 277:32923-32929.

46. Petrucco S, Bolchi A, Foroni C, Percudani R, Rossi GL, Ottonello S: A maize gene encoding an NADPH binding enzyme highly homologous to isoflavone reductases is activated in response to sulfur starvation. Plant Cell 1996, 8:69-80.

47. Braz AS, Finnegan J, Waterhouse P, Margis R: A plant orthologue of RNase $L$ inhibitor (RLI) is induced in plants showing RNA interference. J Mol Evol 2004, 59:20-30.

48. Purdue University Selaginella Page [http://research.e-enter prise.purdue.edu/selaginella]

49. Edwards K, Johnstone C, Thompson C: A simple and rapid method for the preparation of plant genomic DNA for PCR analysis. Nucleic Acids Res 1991, 19:1349. http.//www.biomedcentral.com/1471-2164/6/85 